\title{
Synthesis, Characterization and Antimicrobial activity of 2- Amino-1,3-benzothiazole, Schiff bases and Azo dyes of 2-Amino- 1,3-benzothiazole.
}

\author{
A.G. Awale ${ }^{1}$, Dr. S. B. Gholse ${ }^{2}$, Dr. P.S.Utale ${ }^{3}$ \\ $\left({ }^{1}\right.$ Department of Chemistry, Laxminarayan Institute of Technology, Nagpur, India ) \\ ( ${ }^{2}$ Department of Chemistry, Laxminarayan Institute of Technology, Nagpur, India ) \\ ( ${ }^{3}$ Department of Chemistry, Science College, Nagpur. Maharashtra, India., India )
}

\begin{abstract}
Amino-1,3-benzothiazole was synthesized and schiff bases were prepared by reacting a series of aromatic aldehydes with 2-amino-1,3-benzothiazole to form a number of potentially biologically active compounds.

Azo dyes of 2-amino-1,3-benzothiazole were synthesized by reacting various aromatic amines, substituted amines and phenols.

The structures of 2-amino-1,3-benzothiazole, Schiff bases and azo dyes were confirmed by using $F T-I R,{ }^{1} H$ NMR, Mass, UV, spectroscopic technique.

The dyeing ability of azo dyes was checked by applying azo dyes on various fabrics.

The tiltle compounds were also tested against representatives of gram-positive and gram-negative bacteria by agar diffusion method. Ampicillin was used as positive control.

Key-words : Benzothiazole, aldehydes, schiffs bases, azo dyes, antimicrobial
\end{abstract}

\section{Introduction :}

The twentieth century has been characterized both by a drastic reduction in the mortality caused by infection diseases and by a rise in the control of neoplastic pathologies. Nevertheless, micro-organisms and viruses, on the other hand and tumours on the other still represent a dreadful menace to men's health and therefore, for a more efficient control, require the steady development of novel and more powerful drugs.

It is known that schiff bases possesses biological activity such as antimicrobial, antifungal, antitumor, anti-HIV and herbicides. So we thought to prepare schiffs bases of 2-amino-1,3-benzothiazole and test their biological activity.

The present world is very much fascinated by the different and brilliant colors in every-day life. The colors find a great importance in men's life in various fields such as cloths, food colors, attractive furniture,etc. In biology for staining purpose and in analytical chemistry as an indicators for various titrations. So, we tried to synthesise azo dyes of 2-amino-1,3-benzothiazole.

i. Schiffs bases were prepared by condensing 2-aminobenzothiazole with series of aromatic aldehydes in ethanol. The products obtained were recrystallized from ethanol. The purity and structure of compounds prepared were confirmed by TLC, M.P. and spectral studies.

ii. Azo dyes were prepared by diazotization of 2-amino-1,3-benzothiazole with $\mathrm{NaNO}_{2}$ and $\mathrm{H}_{2} \mathrm{SO}_{4}$ followed by coupling with series of aromatic amines and phenols. Dyes obtained were recrystallized from acetoneethanol mixture. The purity and structures were confirmed by TLC, M.P. and spectral analysis. Dyeing ability of the said compounds was tested by dyeing on various fabrics using standard dyeing method. Dyes synthesised tested as an indicator for acid-base titrations and complexometric titrations.

iii. All these compounds prepared were screened against human pathogenic bacteria. 
1.<smiles>Nc1nc2ccccc2s1</smiles>

3.<smiles>[Al]NNc1nc2ccccc2s1</smiles>

2<smiles>[Al]C=Nc1nc2ccccc2s1</smiles>

2a $\mathrm{Ar}=\mathrm{C}_{\mathrm{B}^{2}}$ $2 \mathrm{~b} \cdot \mathrm{Ar}=4-\mathrm{OCH}_{3} \mathrm{C}_{6} \mathrm{H}_{4}$

2c. $\mathrm{Ar}=2-\mathrm{OHC}_{6} \mathrm{H}_{4}$

$2 \mathrm{e} \cdot \mathrm{Ar}=4-\mathrm{ClC}_{6} \mathrm{H}_{4}$

$2 \mathrm{~g} \cdot \mathrm{Ar}=4-\mathrm{BrC}_{6} \mathrm{H}_{4}$

$2 d \cdot A r=$

2f. $\mathrm{Ar}=$ 4- $\mathrm{NO}_{2} \mathrm{C}_{6} \mathrm{H}_{4}$

$2 \mathrm{~h} \cdot \mathrm{Ar}=4-\mathrm{N}\left(\mathrm{CH}_{3}\right)_{2} \mathrm{C}_{6} \mathrm{H}_{4}$

3. i- ix

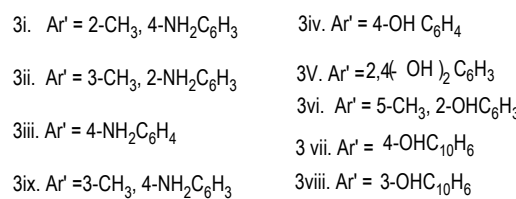

II. Chemistry.

1 2-amino-1,3-benzothiazole is prepared by the method outlined in scheme -I. Cyclization of phenylthiourea was carried out in presence of $\mathrm{Br}_{2}$ as well as $\mathrm{I}_{2}$. The product obtained was recrstallised from 50 $\%$ alcohol and vacuum dried. The test compound had constant in agreement with the reported literature values. The structures confirmed by Mass, IR, NMR, etc.

2. The general method which is employed to prepare schiffs bases ( 2.a-h) is outlined in scheme -II. Desired schiffs bases were prepared by reacting 2 -amino-1,3-benzothiazole with selected aldehydes. The nucleophilic addition of $-\mathrm{NH}_{2}$ group of hetero to aromatic aldehydes is not so straight forward and easier due to the presence of $\mathrm{N}$ in heterocyclic ring. So different conditions were used to synthesis the target compounds eg. Azeotropic distillation method, using dehydrating agents, use of catalysts, etc. All compounds ( a- h) were purified by recrystallization using absolute ethanol in order to avoid hydrolysis at varying rates of the compounds.
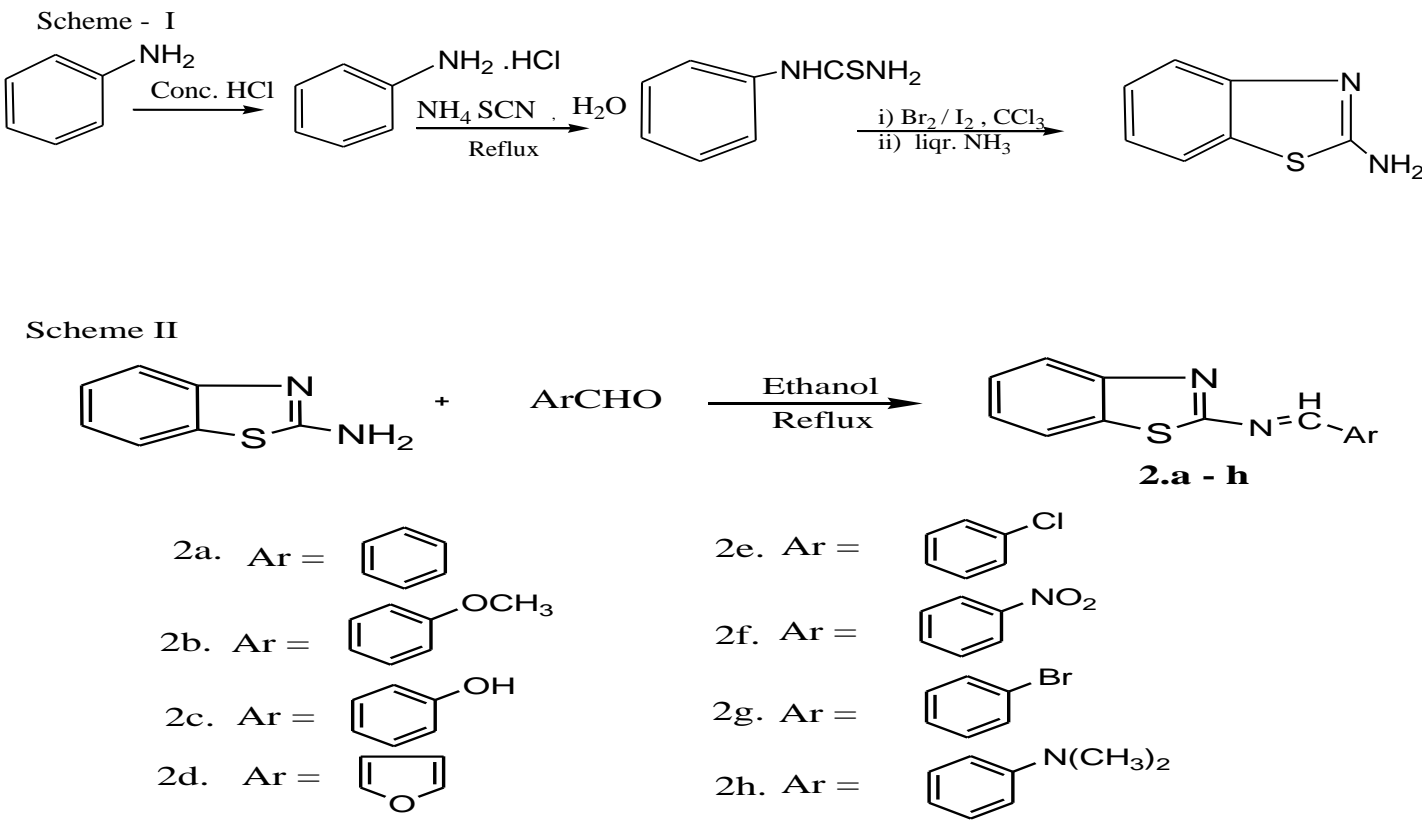
Scheme- III

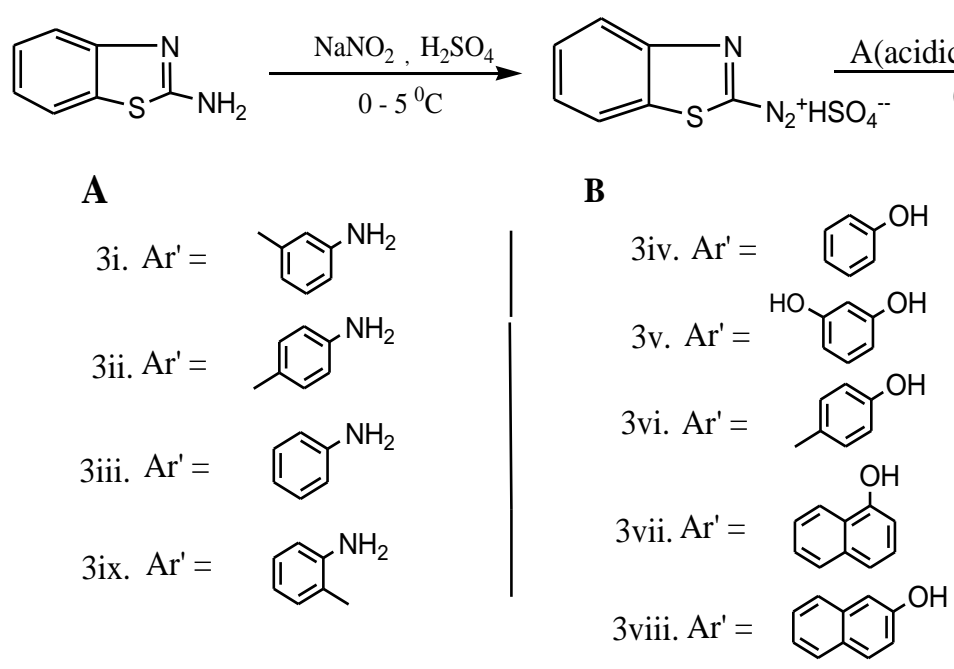

III. Results And Discussions :

The structures of newly described compounds were confirmed by FT-IR , ${ }^{1} \mathrm{H}-\mathrm{NMR}$, LCMS spectroscopic methods. Physical, chemical, IR, NMR, Mass relevant data of the compounds are reported in table no.3.1. The newly synthesized compounds were tested against representatives of gram-positive and gramnegative bacteria ( staphylococcus aureus and Basillus subtilis. ) by agar diffusion method. Ampicillin was used as control. Table no-3.2 shows the Minimum Inhibitory Concentration of Compound (a-h)

Table 3.1. Physicochemical data of benzothiazole Schiff bases.

\begin{tabular}{|c|c|c|c|c|c|c|c|c|}
\hline \multirow{2}{*}{$\begin{array}{l}\text { Compd } \\
\text {. }\end{array}$} & \multirow{2}{*}{$\begin{array}{l}\text { Mol.formul } \\
\text { a. }\end{array}$} & \multirow{2}{*}{$\begin{array}{l}\text { Mol. } \\
\text { Mass }\end{array}$} & \multirow{2}{*}{$\begin{array}{l}\text { M.P. } \\
\left({ }^{\circ} \mathrm{C}\right)\end{array}$} & \multirow{2}{*}{$\begin{array}{l}\text { Yield } \\
(\%)\end{array}$} & \multicolumn{4}{|c|}{$\operatorname{IR} \quad v\left(\mathrm{~cm}^{-1}\right)$} \\
\hline & & & & & $\mathrm{N}=\mathrm{C}$ & $\begin{array}{l}\text { Azomethine } \\
-\mathrm{CH}=\mathrm{N}-\end{array}$ & $\begin{array}{l}\text { Ar.C-H } \\
\text { Str. }\end{array}$ & Substituents \\
\hline $\mathrm{a}$ & $\mathrm{C}_{14} \mathrm{H}_{10} \mathrm{~N}_{2} \mathrm{~S}$ & 238 & 122 & 56 & 1586.90 & $\begin{array}{l}2942,1283, \\
1063\end{array}$ & 3073.18 & -- \\
\hline $\mathrm{b}$ & $\begin{array}{l}\mathrm{C}_{15} \mathrm{H}_{12} \mathrm{~N}_{2} \mathrm{~S} \\
\mathrm{O}\end{array}$ & 268 & 180 & 62 & 1594.63 & $\begin{array}{l}2945, \\
1252.65,1019 \\
.99\end{array}$ & 3184 & $3298.35\left(\mathrm{OCH}_{3}\right)$ \\
\hline $\mathrm{c}$ & $\begin{array}{l}\mathrm{C}_{14} \mathrm{H}_{10} \mathrm{~N}_{2} \mathrm{~S} \\
\mathrm{O}\end{array}$ & 254 & 135 & 58 & 1607.48 & $\begin{array}{l}2920,1282.35 \\
, 1064.40\end{array}$ & 3020 & $3693.75(\mathrm{ArOH})$ \\
\hline $\mathrm{d}$ & $\mathrm{C}_{12} \mathrm{H}_{8} \mathrm{~N}_{2} \mathrm{SO}$ & 228 & 192 & 65 & 1520.00 & $\begin{array}{l}2942,1286, \\
1098\end{array}$ & 3187.09 & -- \\
\hline $\mathrm{e}$ & $\mathrm{C}_{14} \mathrm{H}_{9} \mathrm{~N}_{2} \mathrm{SCl}$ & 273 & 120 & 70 & 1524.72 & $\begin{array}{l}2954.2, \\
1289.01,1089\end{array}$ & 3069.40 & 749.97( C-Cl) \\
\hline $\mathrm{f}$ & $\begin{array}{l}\mathrm{C}_{14} \mathrm{H}_{9} \mathrm{~N}_{3} \mathrm{SO} \\
2\end{array}$ & 283 & 145 & 65 & 1612.16 & $\begin{array}{l}2948,1248, \\
1062\end{array}$ & 3185.35 & $1345.39\left(\mathrm{ArNO}_{2}\right)$ \\
\hline $\mathrm{g}$ & $\mathrm{C}_{14} \mathrm{H}_{9} \mathrm{~N}_{2} \mathrm{SBr}$ & 317 & 190 & 60 & 1524.00 & $\begin{array}{l}2969,1281, \\
1063\end{array}$ & 3085 & $668.05(\mathrm{C}-\mathrm{Br})$ \\
\hline $\mathrm{h}$ & $\mathrm{C}_{16} \mathrm{H}_{15} \mathrm{~N}_{3} \mathrm{~S}$ & 281 & 208 & 70 & 1577.78 & $\begin{array}{l}2950,1255.61 \\
, 1010\end{array}$ & 3000 & $\begin{array}{l}2866.52- \\
\mathrm{N}\left(\mathrm{CH}_{3}\right)_{2,} \\
2966.48\left(\mathrm{CH}_{3}\right)\end{array}$ \\
\hline
\end{tabular}


Table-3.2. Minimum Inhibitory Conecentration of Compound (a-h) ; zone inhibition diameter in $(\mathrm{cm})$

\begin{tabular}{|c|c|c|c|c|c|c|}
\hline \multirow{2}{*}{ Compound } & \multicolumn{3}{|c|}{ Basillus subtilis } & \multicolumn{3}{c|}{ Staphylococcus aureus } \\
\cline { 2 - 7 } & \multicolumn{3}{|c|}{ Concentration $(\mu \mathrm{g} / \mathrm{ml})$} & \multicolumn{3}{c|}{ Concentration $(\mu \mathrm{g} / \mathrm{ml})$} \\
\cline { 2 - 7 } & 50 & 100 & 200 & 50 & 100 & 200 \\
\hline $\mathrm{a}$ & 1.8 & 2.1 & 2.4 & 1.3 & 1.2 & 1.7 \\
\hline $\mathrm{b}$ & 1.1 & 1.2 & 1.5 & 1.1 & 1.6 & 1.3 \\
\hline $\mathrm{c}$ & 1.0 & 1.4 & 1.6 & 0.8 & 1.0 & 1.2 \\
\hline $\mathrm{d}$ & 1.2 & 1.2 & 1.6 & 1.0 & 1.1 & 1.0 \\
\hline $\mathrm{e}$ & 1.5 & 1.5 & 1.8 & 1.1 & 1.0 & 1.0 \\
\hline $\mathrm{f}$ & 1.0 & 0.9 & 1.3 & 0.9 & 0.8 & 1.2 \\
\hline $\mathrm{g}$ & 1.2 & 1.0 & 0.8 & 1.0 & 0.9 & 1.2 \\
\hline $\mathrm{h}$ & 0.9 & 1.0 & 1.0 & 1.1 & 1.0 & 1.3 \\
\hline
\end{tabular}

Ampicillin was used as a positive control. It has been found that all the compounds (a-h) shows good antibacterial activity when compared with ampicillin as a positive control.

\subsection{Azo Dyes Chemistry}

3. The general method which employed to prepare azo dyes of 2- amino-1,3-benzothiazole is outlined in scheme-III. Hetero amine was diazotized and the resulting diazonium salt of hetero amine was treated with various aromatic primary amines and phenols. Azodyes were isolated at $\mathrm{pH}$ 7.0-7.5, recrystallized and air dried. As most of the azo dyes have dyeing ability, so their dyeing ability was tested using various fabrics. The prepared dyes also tested as indicators in various titrations.

Results and discussions :

The structures of newly described compounds were confirmed by FT-IR, H-NMR, LCMS spectroscopic methods. Physical, chemical, IR, NMR, Mass relevant data of the compounds are reported in table no. 3.1.1

Table 3.1.1. Physicochemical data of benzothiazole azo dyes.

\begin{tabular}{|c|c|c|c|c|c|c|c|c|}
\hline \multirow{2}{*}{$\begin{array}{l}\text { Comp } \\
\text { d. }\end{array}$} & \multirow{2}{*}{$\begin{array}{l}\text { Mol.formul } \\
\text { a. }\end{array}$} & \multirow{2}{*}{$\begin{array}{l}\text { Mol. } \\
\text { Mass }\end{array}$} & \multirow{2}{*}{$\begin{array}{l}\text { M.P. } \\
\left({ }^{\circ} \mathrm{C}\right)\end{array}$} & \multirow{2}{*}{$\begin{array}{l}\text { Yiel } \\
\text { d } \\
(\%)\end{array}$} & \multirow{2}{*}{$\begin{array}{l}\text { Rf } \\
\text { valu } \\
\text { e }\end{array}$} & \multicolumn{3}{|c|}{ IR $\quad v\left(\mathrm{~cm}^{-1}\right)$} \\
\hline & & & & & & $\mathrm{N}=\mathrm{N}$ & $\begin{array}{l}\mathrm{C}=\mathrm{N} \\
\text { Thiazol } \\
\mathrm{e}\end{array}$ & Substituents \\
\hline $\mathrm{i}$ & $\mathrm{C}_{14} \mathrm{H}_{12} \mathrm{~N}_{4} \mathrm{~S}$ & 268 & 195 & 40 & 0.6 & 1542.12 & 1607.48 & $\begin{array}{l}3316,3180 \mathrm{~N}-\mathrm{H}, 3059 \mathrm{C}-\mathrm{H}, \\
857-667 \mathrm{Ar} \text { def }\end{array}$ \\
\hline ii & $\mathrm{C}_{14} \mathrm{H}_{12} \mathrm{~N}_{4} \mathrm{~S}$ & 268 & 98 & 50 & 0.5 & 1410.94 & 1558 & $\begin{array}{l}\text { 3174.07 N-H, 3086.70 C-H, } \\
\text { 763.18- 632.22Ar def }\end{array}$ \\
\hline iii & $\mathrm{C}_{13} \mathrm{H}_{10} \mathrm{~N}_{4} \mathrm{~S}$ & 254 & 152 & 48 & 0.5 & 1646.85 & 1603.02 & $\begin{array}{l}3348.05,3211.05 \\
\text { 3000C-H, } \\
\text { 889.90- 631Ar def }\end{array}$ \\
\hline iv & $\begin{array}{l}\mathrm{C}_{13} \mathrm{H}_{9} \mathrm{~N}_{3} \mathrm{~S} \\
\mathrm{O}\end{array}$ & 255 & 240 & 39 & 0.4 & 1465.73 & 1603.91 & $\begin{array}{l}\text { 3631.30 O-H, 3055.67 C-H, } \\
\text { 839.05- 722.95Ar def }\end{array}$ \\
\hline $\mathrm{v}$ & $\begin{array}{l}\mathrm{C}_{13} \mathrm{H}_{9} \mathrm{~N}_{3} \mathrm{~S} \\
\mathrm{O}_{2}\end{array}$ & 271 & 310 & 52 & 0.2 & 1454.97 & 1599.12 & $\begin{array}{l}3239.71 \mathrm{O}-\mathrm{H}, 2927.33 \\
\mathrm{C}-\mathrm{H}, 843.11-624.20 \mathrm{Ar} \text { def }\end{array}$ \\
\hline vi & $\begin{array}{l}\mathrm{C}_{14} \mathrm{H}_{11} \mathrm{~N}_{3} \mathrm{~S} \\
\mathrm{O}\end{array}$ & 269 & 130 & 50 & 0.3 & 1487.83 & 1578.24 & $\begin{array}{l}\text { 3464.52 O-H, } 3000 \mathrm{C}-\mathrm{H} \\
\text { 881.84- } 721.31 \mathrm{Ar} \text { def }\end{array}$ \\
\hline vii & $\begin{array}{l}\mathrm{C}_{17} \mathrm{H}_{11} \mathrm{~N}_{3} \mathrm{~S} \\
\mathrm{O}\end{array}$ & 305 & 240 & 53 & 0.4 & 1455.80 & 1595.47 & $\begin{array}{l}\text { 3296.35 O-H, 3050 C-H, } \\
\text { 874.62- 624.30Ar def }\end{array}$ \\
\hline viii & $\begin{array}{l}\mathrm{C}_{17} \mathrm{H}_{11} \mathrm{~N}_{3} \mathrm{~S} \\
\mathrm{O}\end{array}$ & 305 & 160 & 51 & 0.4 & 1462.19 & 1596.80 & $\begin{array}{l}3273.03 \mathrm{O}-\mathrm{H}, 3052 \mathrm{C}-\mathrm{H} \\
846.61-618.65 \mathrm{Ar} \mathrm{def}\end{array}$ \\
\hline vix & $\mathrm{C}_{14} \mathrm{H}_{12} \mathrm{~N}_{4} \mathrm{~S}$ & 268 & 270 & 47 & 0.3 & 1438.64 & 1596.66 & $\begin{array}{l}\text { 3631.64,3464.10 N-H, } \\
\text { 3059.92,C-H, 822- } 614 \mathrm{Ar} \text { def }\end{array}$ \\
\hline
\end{tabular}

The newly synthesized compounds were tested against representatives of gram-positive and gram- negative bacteria ( staphylococcus aureus and Basillus subtilis ) by agar diffusion method. Ampicillin was used as control. Table no-3.1.2. shows the Minimum Inhibitory Concentration of Compound (i-ix) 
Table-3.1.2. Minimum Inhibitory Conecentration of Compound ( i-ix); zone inhibition diameter in $(\mathrm{cm})$

\begin{tabular}{|c|c|c|c|c|c|c|}
\hline \multirow{2}{*}{ Compound } & \multicolumn{3}{|c|}{ Basillus subtilis } & \multicolumn{3}{c|}{ Staphylococcus aureus } \\
\cline { 2 - 7 } & \multicolumn{3}{|c|}{ Concentration $(\mu \mathrm{g} / \mathrm{ml})$} & \multicolumn{3}{c|}{ Concentration $(\mu \mathrm{gg} / \mathrm{ml})$} \\
\cline { 2 - 7 } & 50 & 100 & 200 & 50 & 100 & 200 \\
\hline i & 0.9 & 0.8 & 1.0 & 1.1 & 1.2 & 1.5 \\
\hline ii & 1.7 & 1.7 & 1.8 & 1.3 & 1.3 & 0.9 \\
\hline iii & 0.8 & 1.1 & 0.8 & 1.5 & 1.8 & 1.9 \\
\hline iv & 1.7 & 1.4 & 1.5 & 1.7 & 1.5 & 1.5 \\
\hline v & 1.2 & 1.4 & 1.1 & 2.0 & 1.5 & 2.1 \\
\hline vi & 1.2 & 1.0 & 0.8 & 1.2 & 1.0 & 0.9 \\
\hline vii & 1.4 & 1.3 & 1.6 & 1.3 & 0.9 & 1.5 \\
\hline viii & 0.9 & 1.0 & 1.1 & 1.5 & 1.6 & 1.6 \\
\hline ix & 1.5 & 1.5 & 1.6 & 1.2 & 1.5 & 1.3 \\
\hline
\end{tabular}

Ampicillin was used as a positive control. It has been found that all the compounds (i-viii) shows good antibacterial activity when compared with ampicillin as a positive control.

It has been found that all the azo dyes (3.i-ix) showed good dyeing ability for cotton fabric. It gives a bright brilliant shades. The fastness to light, hot press and wash (AATCC wash test) are good. The dyeing are dischargeable . it s highly resistant to light and acid fading.

Azo dyes prepared can also be used as an indicators for various titrations such as acid-base titrations and complexometric titrations. They can also be used for staining purpose in biology.

\subsection{Chemistry:}

\section{Experimental :}

Melting points in $\left({ }^{0} \mathrm{C}\right)$ of all Schiff bases are correct and that of all azo dyes are in approximate range. The new compounds were analysed in analytical laboratory of SAIF; IIT ,Bombay, India.

IR spectra were recorded as $\mathrm{KBr}$ pellets, on a FT-IR BRUKER ALPHA-200455. Spectral IR data were consistent with the assigned structure in all cases and the reported wave numbers are given in $\mathrm{cm}^{-1}$

${ }^{1} \mathrm{H}$ NMR spectra in $\mathrm{CDCl}_{3}$ solution were recorded on a Bruker AC300 instrument at 298K. Chemical shifts are reported as $\delta$ ppm relative to TMS as internal standard.

UV-Visible spectra in methanol were recorded on a SHIMADZU SPECTROPHOTOMETER UV-1800, KYOTO ,JAPAN instrument at $298 \mathrm{~K}$.

The progress of the reaction was monitored by Thin layer chromatography with F254 silica gel precoated sheets( MERCK) using pet. ether / ethyl acetate 55/45 as eluent; uv light was used for detection.

Solvents unless otherwise specified, were of analytical reagent grade or of the highest quality commercially available.

\subsubsection{General Procedure For The Synthesis Of 2-Amino-1,3-Benzothiazole}

Aniline Was Treated With Conc. Hcl To Get Water Soluble Salt Of Aniline .

The salt of aniline was dissolved in water and a solution of ammonium thiocyanate added to it. The reaction mixture was boiled for one hour and then poured in ice cold water. White crystals of phenylthiourea obtained was filtered, washed and vacuum dried.

The phenylthiourea was treated with iodine or bromine in chloroform and stirred for four hours at lower temperature. After completion of reaction, the reaction mixture was basified with liquor ammonia. A solid product of 2-amino-1,3-benzothiazole obtained was filtered, washed, recrystallised from 50\% ethanol and vacuum dried.

Experimental data of 2-amino-1,3-benzothiazole :

M.P. $126-128{ }^{0} \mathrm{C}$, mass $[\mathrm{M} / \mathrm{Z}$ (rel. Int )] , 150 (100), 123, 95, 69,

IR-KBr $v\left(\mathrm{~cm}^{-1}\right) .3399-3274 \mathrm{~cm}^{-1}$

$1^{0}$ amine N-H str , $3037 \mathrm{~cm}^{-1}$ ArC-H Str, $1590 \mathrm{~cm}^{-1} \mathrm{C}=\mathrm{N}$ thiazole, $1646-1648 \mathrm{~cm}^{-1}$ ArC $=\mathrm{C}$ str.

${ }^{1} \mathrm{H} \mathrm{NMR}, 300 \mathrm{MHz}, \mathrm{CDCl}_{3}$ Ar- $\mathrm{NH}_{2} \delta 5.8(\mathrm{~s}), \mathrm{Ar}-\mathrm{H} \delta$ 7-7.7(m).

\subsubsection{General Procedure For The Synthesis Of Schiff Bases ( 2.a-h ).}

A solution of 2-amino-1,3-benzothiazole $(5 \mathrm{mmol})$ and appropriate substituted benzaldehyde (6 $\mathrm{mmol}$ ) in $20 \mathrm{ml}$ absolute ethanol with a few drops of glacial acetic acid was refluxed for 7-8 hrs.

The progress of the reaction was monitored by TLC. After completion of reaction, maximum solvent was distilled out and reaction mixture cooled to room temperature. Cold reaction mixture was poured in crushed ice with constant stirring, a precipitate obtained was filtered, washed and recrystallised from alcohol.

Experimental data of Schiff bases of 2-amino-1,3-benzothiazole (2a-h). 
2a. Mass $\left[\mathrm{M} / \mathrm{Z}\right.$ (rel.Int )], $238 .{ }^{1} \mathrm{H}$ NMR, $\left(300 \mathrm{MHz}, \mathrm{CDCl}_{3}, \delta \mathrm{ppm}\right) 10.0$ ( s, $1 \mathrm{H}$,azomethine $\left.-\mathrm{CH}=\mathrm{N}-\right)$, 7.2-8.0 ( $\mathrm{m}, 10 \mathrm{H}$, aromatic $\mathrm{C}-\mathrm{H})$.

2b. Mass [M/Z (rel.Int )], 268 . ${ }^{1} \mathrm{H}$ NMR, $\left(300 \mathrm{MHz}, \mathrm{CDCl}_{3}, \delta \mathrm{ppm}\right) 9.9$ ( $\mathrm{s}, 1 \mathrm{H}$,azomethine $\left.-\mathrm{CH}=\mathrm{N}-\right)$ $3.9\left(\mathrm{~s}, 3 \mathrm{H}, \mathrm{OCH}_{3}\right), 7 .-7.3(\mathrm{~m}, 4 \mathrm{H}$, aromatic $\mathrm{C}-\mathrm{H}), 7.5(\mathrm{~d}, 2 \mathrm{H}, \mathrm{ArC}-\mathrm{H}), 7.9(\mathrm{~d}, 2 \mathrm{H}, \mathrm{ArC}-\mathrm{H})$,

2c. Mass [M/Z (rel.Int )], 254.8. ${ }^{1} \mathrm{H} \mathrm{NMR},\left(300 \mathrm{MHz}, \mathrm{CDCl}_{3}, \delta \mathrm{ppm}\right) 9.4(\mathrm{~s}, 1 \mathrm{H}$,azomethine-CH=N-) $12.2(\mathrm{~s}, 1 \mathrm{H}, \mathrm{PhO}-\mathrm{H}), 7 .-8.0(\mathrm{~m}, 8 \mathrm{H} \mathrm{ArC}-\mathrm{H})$

2d. Mass [M/Z (rel.Int )] , 228. ${ }^{1} \mathrm{H}$ NMR, $\left(300 \mathrm{MHz}, \mathrm{CDCl}_{3}, \delta \mathrm{ppm}\right) 8.1$ ( $\mathrm{s}, 1 \mathrm{H}$, azomethine $\left.-\mathrm{CH}=\mathrm{N}-\right)$ 7.5.-7.7 (m, 4H ArC-H); 7.6 (d, 1H,OC-H), 7.5( d, 1H, fuC-H), 7.2(t, 1H, fuC-H).

2e. Mass [M/Z (rel.Int )], 272.57. ${ }^{1} \mathrm{H}$ NMR, $\left(300 \mathrm{MHz}, \mathrm{CDCl}_{3}, \delta \mathrm{ppm}\right) 10.0(\mathrm{~s}, 1 \mathrm{H}$,azomethine $-\mathrm{CH}=\mathrm{N}-)$ $8.2(\mathrm{dd}, 4 \mathrm{H}, \mathrm{ArC}-\mathrm{H}), 7.5(\mathrm{~m}, 4 \mathrm{H} \mathrm{ArC}-\mathrm{H})$

2f. Mass [M/Z (rel.Int )], 283. ${ }^{1} \mathrm{H}$ NMR, $\left(300 \mathrm{MHz}, \mathrm{CDCl}_{3}, \delta \mathrm{ppm}\right) 10.1$ ( $\mathrm{s}, 1 \mathrm{H}$, azomethine $\left.-\mathrm{CH}=\mathrm{N}-\right)$ 8.1 ( dd, 2H, ArC-H), 8.4 ( dd, 2H, ArC-H), 7.0-7.5 (m, 4H ArC-H)

2g. Mass [M/Z (rel.Int )], 317. ${ }^{1} \mathrm{H}$ NMR, $\left(300 \mathrm{MHz}, \mathrm{CDCl}_{3}, \delta \mathrm{ppm}\right) 10.0(\mathrm{~s}, 1 \mathrm{H}$, azomethine $-\mathrm{CH}=\mathrm{N}-)$ $8.0(\mathrm{dd}, 2 \mathrm{H}, \mathrm{ArC}-\mathrm{H}), 7.9$ ( dd, $2 \mathrm{H}, \mathrm{ArC}-\mathrm{H}), 7.7$ (m, 4H ArC-H)

2h. Mass [M/Z (rel.Int )], 282.2. ${ }^{1} \mathrm{H} \mathrm{NMR},\left(300 \mathrm{MHz}, \mathrm{CDCl}_{3}, \delta \mathrm{ppm}\right) 8.9$ ( $\mathrm{s}, 1 \mathrm{H}$, azomethine $\left.-\mathrm{CH}=\mathrm{N}-\right)$ $\left(\mathrm{s}, 6 \mathrm{H},-\mathrm{N}\left(\mathrm{CH}_{3}\right)_{2}\right), 7.8$ ( d, 2H, ArC-H), 7.9 ( d, 2H, ArC-H), 7.2-7.6 (m, 4H ArC-H)

\subsubsection{General Procedure For Synthesis Of Azo Dyes Of 2-Amino-1,3-Benzothiazole (3. i - ix )}

Azo dyes of heterocyclic amines ( $\mathrm{i}$ - ix ) were prepared by diazotization of 2-amino-1,3benzothiazole with $\mathrm{NaNO}_{2}$ and $\mathrm{H}_{2} \mathrm{SO}_{4}$ at $0-5{ }^{\circ} \mathrm{c}$ followed by coupling with series of aromatic amines and phenols at temperature below $5{ }^{\circ} \mathrm{c}$, the reaction mixture was stirred for 3-4 hrs and then at RT for $1 \mathrm{hr}$. The reaction mixture was then treated with sodium acetate solution to make $\mathrm{pH}$ 7-8. Products obtained were filtered, washed and recrystallized from acetone- ethanol mixture and air dried.

\section{Experimental Data Of Azo Dyes Of 2- Amino-1,3-Benzothiazole (3.I-Ix)}

3. i. Mass [M/Z (rel.Int )] ,269.151,124,106. ${ }^{1} \mathrm{H}$ NMR, $(300 \mathrm{MHz}) ,2.2\left(\mathrm{~s}, 3 \mathrm{H}, \mathrm{ArCH}_{3}\right), 2.8\left(\mathrm{~s}, 2 \mathrm{H}, \mathrm{ArNH}_{2}\right)$

7.4-7.6( m,3H,ArC-H), 7.8-8.2( d,d,4H,Ar'C-H). UV- $\lambda \max (\mathrm{nm})$. 528.84

3.ii. Mass [M/Z (rel.Int )], 269.15. ${ }^{1} \mathrm{H} \mathrm{NMR},(300 \mathrm{MHz}) ,2.4\left(\mathrm{~s}, 3 \mathrm{H}, \mathrm{ArCH}_{3}\right), 2.6\left(\mathrm{~s}, 2 \mathrm{H}, \mathrm{ArNH}_{2}\right)$

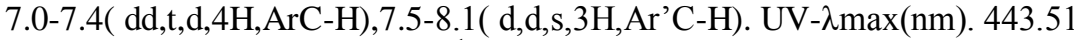

3.iii. Mass [M/Z (rel.Int )], $254{ }^{1} \mathrm{H}$ NMR,(300MHz,) $2.6\left(\mathrm{~s}, 2 \mathrm{H}, \mathrm{ArNH}_{2}\right)$

7.3-7.5( m,4H,ArC-H),6.7-6.8,( d,2H,Ar'C-H), 7.8-8.1(t,d 2H, ArC-H). UV- $\lambda$ max(nm). 487.06

3.iv. Mass [M/Z (rel.Int )] , 254.9,195.1,134. ${ }^{1} \mathrm{H}$ NMR,(300MHz,) 2.8( s ,1H,PhO-H)

7.0-7.5( m,4H,ArC-H), 7.8-8.0(d,d 4H, Ar'C-H). UV- $\lambda \max (\mathrm{nm}) .421 .32$

3.v. Mass [M/Z (rel.Int )], 271. ${ }^{1} \mathrm{H}$ NMR, $(300 \mathrm{MHz}) ,3.9(\mathrm{~s}, 2 \mathrm{H}, \mathrm{PhO}-\mathrm{H})$

7.4-7.8( m,4H,ArC-H), 7.8-8.2(d,d,s,3H, Ar'C-H). UV- $\lambda \max (\mathrm{nm}) .492 .08$

3.vi. Mass [M/Z (rel.Int )], 269. ${ }^{1} \mathrm{H}$ NMR, $(300 \mathrm{MHz}) ,1.6\left(\mathrm{~s}, 3 \mathrm{H}, \mathrm{ArCH}_{3}\right) 2.5(\mathrm{~s}, 1 \mathrm{H}, \mathrm{PhO}-\mathrm{H})$

7.4-7.8( m,4H,ArC-H), 7.0-7.8(d,d,s,3H, Ar'C-H). UV- $\lambda \max (\mathrm{nm}) .422 .17$

3.vii. Mass [M/Z (rel.Int )], 305. ${ }^{1} \mathrm{H}$ NMR, $(300 \mathrm{MHz}) ,2.8(\mathrm{~s}, 1 \mathrm{H}, \mathrm{PhO}-\mathrm{H})$

7.4-8.4( m,6H,Ar'C-H),6.5-7.2(m,4H,ArC-H). UV- $\lambda \max (\mathrm{nm}) .323 .25$

3.viii. Mass [M/Z (rel.Int )], 305. ${ }^{1} \mathrm{H}$ NMR, $(300 \mathrm{MHz}) ,2.5(\mathrm{~s}, 1 \mathrm{H}, \mathrm{PhO}-\mathrm{H})$

7.2-7.4( m,6H,Ar'C-H), 7.6-7.8(m,4H,ArC-H). UV- $\lambda$ max(nm). 330.12

3.ix. Mass [M/Z (rel.Int )], 269.15. ${ }^{1} \mathrm{H}$ NMR, $(300 \mathrm{MHz}) ,2.2\left(\mathrm{~s}, 3 \mathrm{H}, \mathrm{ArCH}_{3}\right), 2.8\left(\mathrm{~s}, 2 \mathrm{H}, \mathrm{ArNH}_{2}\right)$

7.2-7.4( m,4H,ArC-H), 7.8-8.4( d,d,s,3H,Ar'C-H). UV- $\lambda \max (\mathrm{nm}) .351 .92$

\section{Acknowledgements:}

The authors are sincerely thankful to the Director of LIT, Nagpur, India for providing laboratory facilities and the Head SAIF,IIT Bombay, India for providing IR, H1NMR, Mass, UV spectroscopic analytical facility. The authors are also thankful to the Head Dept. Of Polymer and Textile Engineering, UICT, Matunga, Mumbai for constant support in dyeing techniques of fabrics.

[1] Goodwin, jill. A Dyer's manual, Pelham, 1982. P11

[2] Geronikaki, A; Theophilidis, G. Eur. J.Med. Chem. 1992,27,709

[3] Padmavathi P. Prabhu, Sushant Pande, C.S. Shastry, Int. J.Chem. Tech, 2011, 3,1,185

[4] Hugerschoff, H, Chem. Ber. 1901,34,3130, Hugerschoff, H.chem Ber., 1903, 36

[5] Christopher Stanley, Argyle and Sydney Alfred Gilson, U.S.Patent,1948,2441612

[6] Joseph W. Dehn, Jr. Bayside, Englewood, N.J. U.S.Patent, 1962, 3057848

[7] Joachim George, Leverkusen, U.S.Patent, 1981, 4281195

[8] Amany M.A.Ibrahim. Can. J.Chem. 71,318(1993)

[9] Jung MH. Korean J.med. Chem1999;9(2); 56-62

[10] Shreenivas M.T. et al/ J. Pharma. Sci. Tech 1(2), 2009, 88-94 
[11] K.R.H. Wooldrige, Adv. Heterocyclic Chem. , 14,191982).

[12] R.K.Bansal, heterocyclic chem. 5 Edn. 487 (book)

[13] Cristina Cojocarius and Paul Rochon, J. Materials Chem, 2004

[14] Raafat M.Issa, Abdalla m. Khedr, Helen Rizk, J.Chin. Chem.. Soc, 2008,55, 875-884

[15] Zollinger, H (1991): Text chem. Colorist, 23(12), 19

[16] Todor G. Deligeorgiev, dimitar Simov, dyes and pigments,38,1-3,115-125, 1998.

[17] Allen, C.F.H. and Van-Allan, J. Org. Synth, 1942,22,16(b)1955,3,76.

[18 Morlacchi, F.,Armenise, D.,De Laurentis, N.,Reho A. And Rosato,A.,J. Heterocyclic chem.. 2004,41,771.

[19] S. Bondock, W. Fadaly and M.A. Metwally, J. Sulphur chem., 30,1,2009, 74-107

[20] Jolanta Sokolowska-Gajda and Harold S. Freeman, Dyes and pigments ,20(1992)137-145

[21] S.B.Junne et al. (2010) E.Journal of chemistry, 7(3), 882-886 